\title{
Critical and Comprehensive Ethical Analysis on Pragmatic Randomized Controlled Trials
}

\author{
Soaad Q. Hossain \\ Department of Philosophy, Department of Computer and Mathematical Sciences, \\ University of Toronto, Canada
}

\begin{abstract}
The interest of pragmatic randomized controlled trials continues to increase as they are much better suited for studies of how to get medical and health services out into wider practice. However, despite the advantage that such trials have, there are several ethical issues and medical ethics issues that persist with the trial. The ethical and medical ethics issues involve research-practice distinction, consent, disclosure, vulnerable populations, oversight, ethical principles, ethical framework, regulatory frameworks, and conflicts of interest. Through performing an elaborate literature review and analyzing claims and arguments made within the literature, we will provide a critical and comprehensive ethical analysis on pragmatic randomized controlled trials, and we will begin the discussion on conflicts of interest in pragmatic RCTs, arguing that conflicts of interest occur in pragmatic RCTs.
\end{abstract}

Keywords: pragmatic, randomized control trials, ethical, research, practice, distinction, disclosure, harm, principle, risk, consent, oversight, issue, randomization, clinical, vulnerable, participants, framework

\section{Introduction}

The interest for pragmatic randomized controlled trials has and continues to increase within the medical and healthcare sector as they're well suited for studies of how to get medical and health services into practice and can remove information biases due to lack of randomization while still providing evidence that closely captures routine care (Dal-Ré, Janiaud, Loannidis, 2018). As they are conducted in real-world clinical practice settings, not only are can they be used to test interventions, but they can also be used to illustrate the differences in how different types of intervention works in medical and healthcare settings, enabling researchers and practitioners to better understand how different types of intervention works in actual healthcare settings.

While there are benefits with the use of pragmatic randomized controlled trials, there are several major ethical issues that concern pragmatic randomized controlled trials (that exist and are not being properly investigated or being discussed within the scientific community). These major ethical issues stem from research-practice distinction, consent, disclosure, oversight and conflicts of interest. This paper will first provide details about pragmatic randomized controlled trials and how they differ from explanatory randomized controlled trials, followed by a literature review on selected literature that focuses on the ethics of pragmatic randomized controlled trials followed by a critical and comprehensive analysis on the ethical issues highlighted by the literature review and the arguments associated with it. The paper will conclude with the discussion on conflicts of interest in pragmatic RCTs, arguing that conflicts of interest occur in pragmatic RCTs.

\section{Background}

The World Health Organization defines clinical trials as any research study that prospectively assigns individual or groups of human participants to one or more medical or health-related intervention to evaluate the effects on health outcomes. Clinical trials can either be designed to be explanatory or pragmatic. Explanatory trials are trials designed to help determine whether a 
treatment or intervention has any efficacy under ideal, experimental conditions (MacPherson, 2004). Pragmatic trials are trials designed to help determine how effective a treatment or intervention in routines practice (MacPherson, 2004). Explanatory and pragmatic trials are two forms of randomized controlled trials (RCTs). Randomized controlled trials are a type of study design that randomly assigns participants into an experimental group or a control group to test a specific drug, treatment or other intervention to then evaluate a health service delivery or a medical treatment. Pragmatic randomized controlled trials are a type of study design that randomly assigns participants into an experimental group or a control group to test the effectiveness of a specific intervention in usual clinical conditions and day-to-day practice. There are several differences between explanatory RCT and pragmatic RCT; those being (1) the objective of the designs, (2) the selection of the participants, (3) the intervention settings, (4) the delivery of the intervention, and (5) the assessment of the outcomes.

To more easily comprehend the differences between explanatory and pragmatic RCT prior to going into explanations and details about the differences, we will present a simple example of a study on vaccines. In an explanatory RCT, a new vaccine is being studied to see whether the vaccine is effective in treating some disease. In testing the effectiveness of the vaccine, the participants, individuals that met strict eligibility criteria, would be randomly allocated to one of two groups, and the study setting would be controlled so that no interference takes place when conducting the experiments. However, in a pragmatic RCT, rather than a new vaccine being studied, an existing vaccine is being studied to see how effective it is given existing healthcare settings and in routinely clinical practice. In testing the effectiveness of the vaccine, the participants, patients and individuals that did not need to meet strict eligibility criteria, would be randomly allocated to one of two groups, and the study setting would not be controlled so that the setting is as real-world as possible. As it can be seen, there are visible differences between explanatory RCT and pragmatic RCT.

\section{Design Objective}

There are several differences between explanatory RCT and pragmatic RCT. Explanatory RCT aims for causal understanding (Charlton, 1994), and to ensure internal validity; that is, prevention of bias (Dal-Ré, Janiaud, Loannidis, 2018). Pragmatic RCT aims for estimations for comparing the effect of treatments and interventions in practice (Charlton, 1994), and to maximize external validity while preserving as much internal validity as possible; that is, maximizing generalizability of the results to many real-world settings while preserving as much prevention of bias as possible (Dal-Ré, Janiaud, Loannidis, 2018). This differs in understanding because with explanatory RCT, it involves understanding the effectiveness of an intervention that may or may not have been approved. Alternatively, for pragmatic RCT, it involves understanding the effectiveness of an already approved intervention in routinely clinical practice.

\section{Participants Selection}

For explanatory RCT and pragmatic RCT, as both are randomized trials, participants are allocated to a treatment or intervention based on chance rather than being assigned based on eligibility requirements (Sidani, 2015). However, explanatory RCT, participants may be randomized in the order that they are recruited, while for pragmatic RCT, participants are often randomized at a group level. Groups in the sample of pragmatic RCT must be representative of the subgroups forming the target population seen in routines and every practice because it enhances the relevance of the findings to the real world (Sidani, 2015). Researchers conducting explanatory RCT follow strict eligibility requirements when accepting participants for their studies. Consequently, researchers conducting pragmatic RCT do not have exhaustive eligibility requirements when accepting participants for their studies as with using a pragmatic approach, using participants that meet fewer requirements promotes diversity in the participants in effort of having a diverse population for the study. 


\section{Intervention Setting}

The settings in which the intervention is implemented for explanatory RCT and pragmatic RCT are selected are different. For explanatory $\mathrm{RCT}$, the setting is selected through considering environmental features that may affect the accuracy of the intervention being implemented, and the outcome that are produced. The reason for this is because within an environment, there can be certain variables in it that can impact the experiment being conducted in a study. Constant unwanted and disruptive loud noises, for example, is a variable that could hinder the performance of a participant in a psychology study that requires high concentration. The selection of the setting is chosen such that the features of the setting are those that facilitate intervention delivery while also not interfere with the outcome achievement. Also, specific parts of the environment are consistent across all participants receiving the intervention. For pragmatic RCT, a variety of practice settings are selected. The selection of the setting is chosen such that the features of the setting are those that facilitate the accumulation of different subgroups forming the target population in sufficient quantities, and the setting represents contextual factors.

The contextual factors have the potential to influence both the implementation and effectiveness of the intervention. The variability in the setting's features are sought depends on the characteristics of the target population, the intervention, and the extent which environmental features interferes with intervention implementation. To assist with identifying the setting's features that facilitate (or hinder) the effectiveness of the intervention, it is necessary that diversity in contextual features is ensured and examination of their contribution to intervention implementation and outcome achievement takes place (Sidani, 2015).

\section{Intervention Delivery}

Intervention delivery for explanatory RCT and pragmatic RCT heavily differ from one another. In pragmatic RCT, the implementation and delivery of the intervention does not require specialized healthcare professionals to implement and deliver them. This differs from RCT as RCT require a specialized medical or healthcare professional that is well-suited about the intervention to implement and deliver the intervention. This difference is what makes pragmatic RCT more flexible than RCT in terms of intervention delivery (Horn et al., 2018). In using the different approach from that of RCT, pragmatic RCT aims to determine transferability of the intervention to day-to-day practice (Sidani, 2015).

\section{Outcome Assessment}

Assessment of outcomes in explanatory RCT and pragmatic RCT occurs before and after the intervention implementation. For explanatory RCT, the impact of the intervention on a single outcome is evaluated. Optimal empirical evidence of the effects of the intervention is achieved through making measurements of outcomes on participants assigned to the intervention take place before and after the implementation of the intervention and statistical analysis of the outcome data. Outcome analysis in explanatory RCT is focuses on demonstrating statistical significance. For pragmatic RCT, the impact of the intervention on a range of outcomes is evaluated. Outcomes are assessed at regular time intervals, over a prolonged follow-up period, which the time intervals are determined based on the expected pattern of change in the outcome (Sidani, 2015). Outcome analysis in pragmatic RCT is focuses on demonstrating statistical significance and clinical relevance of effects from the intervention.

\section{Literature Review}

Current literature review on the ethics of pragmatic RCTs discuss four major bioethics themes: research-practice distinction, consent, disclosure, and oversight (Goldstein et al., 2018). In addition to the four major bioethics themes, there are three emerging themes being discussed within a subset of the existing literature review. These three emerging themes pertain to how pragmatic are RCTs labeled as 'pragmatic', vulnerable populations in pragmatic RCT, and 
current ethical principles, ethical framework and regulatory frameworks. Within one of the research articles, there has been a mention about conflicts of interest in pragmatic RCT, highlighting that there are no literature discussing of the risk posed by conflicts of interest in the case of commercially sponsored pragmatic RCTs (Goldstein et al., 2018). For that reason, it is also unclear whether there are proper discussions taking place regarding conflicts of interest and pragmatic RCTs within the scientific community. When we say scientific community, we mean solely mean the medical and healthcare community. Thus, while we will be discussing conflicts of interest later, we will not discuss about conflicts of interest in the literature review.

\section{Research-Practice Distinction}

The term "research" refers to a class of activities designed to develop or contribute to theories, principles or relationships that can be corroborated by accepted by accepted scientific observation and inference (Emanuel et al., 2003). The term "practice" of medicine or behavioral therapy refers to a class of activities designed only to enhance the well-being of an individual patient or client (Emanuel et al., 2003). While both terms are distinct in terms of their definition, a central question currently discussed within the scientific community is whether a meaningful distinction exists between research and clinical practice. From the literature on pragmatic RCTs, the majority of authors are critical of the relevance of the researchpractice distinction (Goldstein et al., 2018). A distinction between research and clinical practice is that in terms of participation, research participation involves more risk to patients than clinical practice. For instance, in testing the performance of a drug, there is more risk of harm to the patient in that than in prescribing a drug to the patient. However, authors argue that such distinction is irrelevant in pragmatic RCT because pragmatic RCTs compare treatments used routinely in clinical practice and pose fewer additional risks to participants (Goldstein et al., 2018), thus leading to the assumption that research automatically involves higher risk than clinical practice.

\section{Consent}

The way that consent is seen through authors differs from that of the purpose of consent. In research ethics, the purpose of consent is to provide participants more autonomy, implying that consent is needed as it empowers people by allowing them to make decisions for themselves. This differs from how authors see consent as they see it as they see consent as something that is not necessary as they see it as required only because of the added risks that research poses compared to clinical practice.

From research-practice distinction, given that clinical practice is lower risk that research, authors support simplified consent or no consent for pragmatic RCT. However, there are individuals that support different kinds of consent - those types of consent being streamlined consent, altered consent, integrated consent, and targeted consent. Streamlined consent is a type of consent that takes place inside a set of structures within a learning health care system, which includes ethics oversight panels and public notification, allowing for the elimination of consent in many cases ((Goldstein et al., 2018). Altered consent, also known as simplified consent, is a type of consent that aims to balance the need to obtain informed consent with associated burdens including cost, time, and complexity (Goldstein et al., 2018). Integrated consent is a type of consent that uses verbal communication to consent for research is obtained in a clinical setting. Lastly, target consent is a consent that requires participants to sign a consent form after the discussion on pragmatic RCT participation has taken place.

\section{Disclosure}

Within research using pragmatic RCTs, there are several components within disclosure to consider - those components being the research purpose, the risks and benefits associated with the research, the voluntariness of participation in the research, and randomization associated with pragmatic RCTs. Among the components, not all authors agree to disclosing all the components to the participants or prospective participants. In general, among authors that believe that informed 
consent out to be obtained, there exists disagreements as to what needs to be disclosed to participants in low-risk trials. Furthermore, there is also a disagreement among authors as to whether randomization needs to be disclosed (Goldstein et al., 2018). Authors against disclosing randomization argued that since randomization does not increase risk, disclosing it to participants is not needed. Consequently, authors for disclosing randomization argued that since not disclosing randomization is problematic for both ethical and practical reasons, therefore it should be disclosed to participants (Goldstein et al., 2018).

In disclosing the purpose of the research of the pragmatic RCT to prospective participants, among those that believe that informed consent out to be obtained, there is an agreement that it should be disclosed. In addition, there is also an agreement to disclosing the benefits and risks associated with the pragmatic RCT to prospective participants, and an agreement to disclosing the voluntary nature of participation in pragmatic RCTs to participants and prospective participants (Goldstein et al., 2018).

\section{Research Oversight}

In any research, oversight is conducted by research ethics committees. The research ethics committee is responsible for overseeing research ethics, grants and contracts, conflicts of interest, patient safety, and pharmacy in a study (Goldstein et al., 2018). Research oversight over clinical research is one of the main means of ensuring that human subjects are protected from the natural bias of researchers and research institutions in favor of experimentation (Wenner, 2016). The benefits of well operated oversight are that not only does it protect prospective participants, but it also helps reinforce the trust that the public has in a system of evidence production that depends largely on the ongoing support of stakeholders, which those stakeholders have a wide range of personal motives for contributing to its continued existence (Wenner, 2016).

Despite the benefits that oversight provides, in the case of pragmatic RCTs, authors and even those within the scientific community that conduct pragmatic RCTs find that research oversight are burdensome and are a barrier to conducting pragmatic RCTs due the fact for many researchers, research ethics committees are conceived as causing delays and hindering the overall progress of research with little added value (Goldstein et al., 2018). Researchers find that the review process conducted by research ethics committees for pragmatic RCTs are time consuming, costly, overly complex and lacks standardization.

\section{Pragmatism}

While the first article introducing the concept of pragmatism was published in 1967 , the scientific community has only recently started to be aware of the issue (Patsopoulos, 2011). From 1970 onward, terms like pragmatic and even its synonyms, practical and naturalistic, have been used at an increasing rate to express the need for more evidence that is applicable in day-to-day clinical settings. A study by Nikolaos Patsopoulos investigated the appearance of the word pragmatic or naturalistic in the titles or abstracts of articles indexed in MEDLINE, and the tag clinical trial and randomized controlled trial in articles indexed in MEDLINE. Figure 1 is a diagram from the study showing the increasing use of the word pragmatic or naturalistic in the titles or abstracts of articles indexed in MEDLINE. However, as Patsopoulos mentioned in his study, although the search used to identify the articles in his study is neither sensitive (not all pragmatic trials and articles on the subject are included) nor specific (the retrieved records might not be in fact pragmatic trials or discuss issues on the subject), there is a clear indication that the scientific community is more sensitized to pragmatism, and is being encouraged in the sense of encouraging the increasing rate of clinical trials that use the words pragmatic and naturalistic in the title or the abstract (Patsopoulos, 2011).

Following up with the increasing use of the term pragmatic came discussions of how pragmatic RCTs labeled as 'pragmatic' is currently being discussed among bioethics communities and even some scientific communities. In investigating how pragmatic are randomized controlled trials labeled 


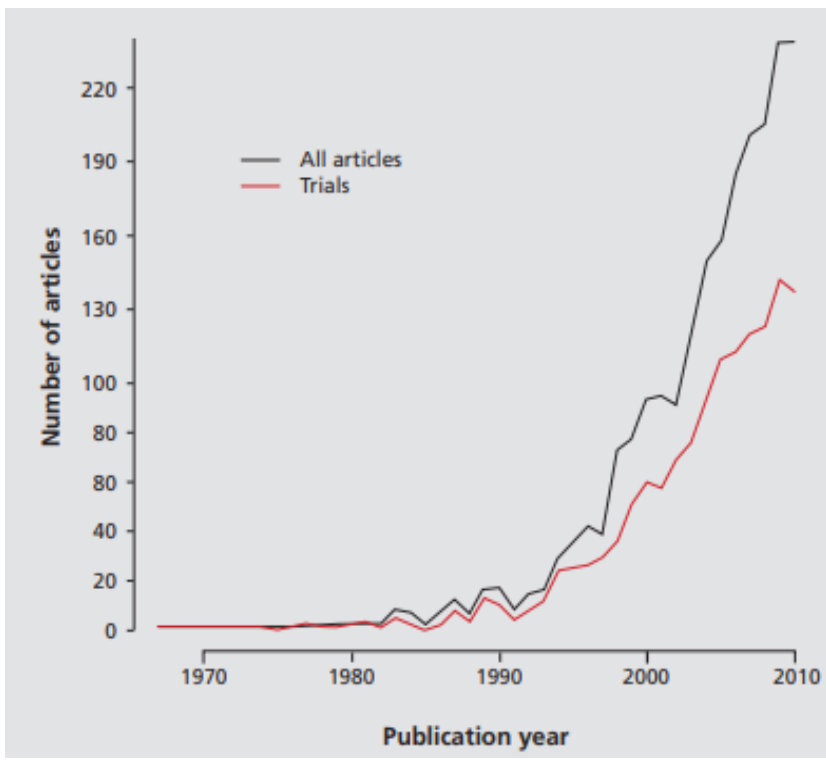

Figure 1: Diagram displaying the articles per year catalogued in MEDLINE that have in the title or abstract the words "pragmatic" or "naturalistic" and the word "trial". The red line represents the articles that are tagged from MEDLINE as "clinical trial" or "randomized controlled trial". Diagram taken from Patsopoulos N. 2011, A Pragmatic View on Pragmatic Trials, Dialogues in Clinical Neuroscience, and accessed on April 16, 2019.

as pragmatic, a study by Rafael Dal-Ré, Perrine Janiaud and John Loannidis found 615 RCTs from 1977 to 2017, self-labeled as pragmatic in their titles, which of the 615 RCTs, 89 RCTs were on medicines. Among the 89 RCTs, 5 RCTs addressed investigational medicines before licensing, 16 RCTs were single-center, 4 RCTs used multiple placebos for blinding, and 14 RCTs used a single placebo (Dal-Ré, Janiaud \& Loannidis, 2018). Pragmatically speaking, as RCTs comparing a single active medicine versus a single (or multiple) placebo cannot be pragmatic, and because the use blinding cannot be pragmatic, this implies that from the study, between 1977 to $2017,36 \%$ of RCTs that were on medicines and labeled as pragmatic were not pragmatic. This finding has led to concerns over the misuse of the word pragmatic. As a result, the study emphasized the need for better standardization of the term 'pragmatic'. In addition, the study also proposed the use and disclosure of the scores produced by the PRECIS2 tool, a tool that incorporates a 10-spoke "wheel" to provide a visual representation that allows investigators to assess the degree to which a trial incorporates "pragmatic" principles (Califf \& Sugarman, 2015), to allow reviewers and editors to better appraise the degree of pragmatism in the RCT, and proposed that the final PRECIS-2 tool assessment agreed between the authors and the journal editor should be published to inform readers for the reasons supporting the use of pragmatic to describe the RCT, and consequently, whether the RCT is gathering real-world evidence (Dal-Ré, Janiaud \& Loannidis, 2018).

\section{Vulnerable Populations}

A person is categorized as vulnerable if they are incapable of protecting their own interests (Welch et al., 2015). The vulnerable population includes those that are children, infants, prisoners, pregnant women, fetuses, neonates, people with physical disabilities or mental illnesses, and disadvantaged people. A primary reason why such people are considered vulnerable is the question of their ability to provide informed consent. To protect vulnerable people in clinical research, policies have been developed. Article 9.2 on the nature and extent of community engagement in the Canadian Tr-Council Policy Statement's (TCPS 2) is an example of a policy that protects vulnerable populations such as Aboriginal people in Canada. Article 9.2 states the following (Government of Canada, 2018):

"The nature and extent of community engagement in a project shall be determined jointly by the researcher and the relevant community and shall be appropriate to community characteristics and the nature of the research."

In having this policy in place, this prevents researchers from taking advantage of vulnerable community members. As research policies have been designed around RCT and not pragmatic RCT, authors, specifically Mary Jane Welch et al., have raised the concern that vulnerable populations participating in pragmatic RCTs are at risk of harm in their article "The Ethical and Regulatory Landscape of Including Vulnerable Populations in Pragmatic Clinical Trials", arguing that protections 
for vulnerable individuals in explanatory RCT settings may not be translatable, feasible, or even ethical to apply in pragmatic RCT, and emphasize that pragmatic RCT should be equally low-risk for all participants (including vulnerable participants) in a pragmatic RCT (Welch et al., 2015). They also argued that pragmatic RCT is designed to make the question about vulnerable people and their ability to provide informed consent less relevant when consent is changed.

These arguments brought forth by authors led them to an ethical argument on inclusion, which argues for inclusion of vulnerable participants in pragmatic RCT. In cases such as that of children and pregnant women, given the lack of data to support the safety and efficacy of treatment in these populations, it is important to allow equitable access to the knowledge gained from research, and to ensure the information learned from pragmatic RCTs is publicly dissimilated to contribute to generalizable knowledge and honor the participation of children and pregnant women participants as well as other vulnerable participants (Welch et al., 2015). Lastly, they discussed how designs for pragmatic RCT should consider inclusion of participants who may be a member of a vulnerable group, and specific considerations for children, disadvantaged persons, human fetuses, the institutionalized, neonates, persons with physical handicaps or mental disabilities, pregnant women, prisoners, racial minorities, and the very sick are should be given to vulnerable participants participating in a pragmatic RCT. For instance, some of those that fall under the vulnerable category of the disadvantaged populations have financial difficulties that make it challenging for them to stay enrolled in a pragmatic RCT. Financial difficulty can cause disadvantaged people to discontinuation their phone services, preventing them from following up with the pragmatic RCT. While discontinuation was used as an example to show how it may be hard for a disadvantaged person to follow through with a pragmatic RCT, there has been reports that discontinuation of phone services is a barrier to long-term follow-ups in pragmatic RCTs (Welch et al., 2015).

\section{Principles and Framework}

Current research ethics is founded upon four internationally accepted ethical principles: respect for individuals, beneficence, justice, and respect for the community (Horn et al., 2018). The ethical principles aim to protect patients, participants and communities. However, authors have highlighted that ethical principles, ethical framework and regulatory frameworks were developed with explanatory RCTs in mind. Knowing the difference between explanatory RCT and pragmatic RCT, authors express major worries that the existing ethical principles, ethical framework and regulatory frameworks will not be able to address ethical issues found in clinical practice. As a result, this could lead to harm to participants participating in pragmatic RCT. Consequently, ethical concern and challenge being debated within the literature is the discussion surrounding the current ethical principles, ethical framework and regulatory frameworks and whether given those principles and the frameworks governing medical and healthcare practice and research are capable of protecting the rights and interests of patients and vulnerable and non-vulnerable research participants while remaining sufficiently flexible to accommodate pragmatic RCT (Califf \& Sugarman, 2015). With there being an absence of clear ethical guidance for pragmatic RCTs, this poses a practical threat to the conduct of the research and increases the risk of harm of participants in pragmatic RCTs.

\section{Comprehensive Ethical Analysis}

From the literature review, there are several ethical issues that have mentioned. In this section, we will analyze and elaborate on those ethical issues and introduce new ethical issues with pragmatic RCT. More specifically, we will provide a critical and comprehensive analysis of the ethical issues in pragmatic RCT with respect to researchpractice distinction, consent, disclosure, oversight, participation, pragmatism and ethical principles, ethical framework and regulatory frameworks, and start the discussion on the [ethical] issue of conflicts of interest in pragmatic RCTs.

\section{Randomization Issues}


One of the most noticeable ethical issues found in pragmatic RCTs are those found in RCTs. With pragmatic RCT being a type of randomized trial, the ethical issues surrounding randomization apply to pragmatic RCT. The main concern with randomization is that patients and participants enrolled in an RCT are used to improve medical knowledge (in pragmatic RCT it would be healthcare knowledge), but they cannot be the beneficiaries of the results of the trials in which they are participating (Colli, Pagliaro \& Duca, 2014). The other ethical issue with randomization comes from disclosure. As this ethical issue and the ethical issues in research-practice distinction are strongly connected, we will discuss and elaborate on this ethical issue after covering the ethical issues found in research-practice distinction, which will be covered in the next section.

\section{Research-Practice Distinction Issues}

When it comes to research-practice distinction and pragmatic RCTs, not only does ethical issues associated with research-practice distinction and pragmatic RCTs exist, but the ethical issues expand to ethical issues in other areas as well. We will first investigate the ethical issues associated with research-practice distinction and pragmatic RCT, then transition to ethical issues associated with research-practice distinction, ethical principles, ethical framework and regulatory frameworks, and move forward with the ethical analysis from there.

The distinction that was mentioned within literature between research and clinical practice is that given that pragmatic RCTs compare treatments used routinely in clinical practice and pose fewer additional risks to participants (compared to explanatory RCTs), pragmatic RCTs are considered low-risk. While those may be true, can we automatically categorize pragmatic RCTs as low-risk trials? We know that RCTs require a specialized medical or healthcare professional that is well-suited about the intervention to implement and deliver the intervention. Consequently, we also know that the implementation and delivery of interventions in a pragmatic RCT do not require specialized medical or healthcare professionals. The ethical issue arises from such difference. In not having specialized medical and healthcare professionals carry out interventions in pragmatic RCTs, participants are at a higher risk of harm because specialized medical or healthcare professionals are better at carrying out interventions than non-specialized medical or healthcare professionals.

Consider the case of neurosurgery involving deep brain stimulation as the surgical intervention. The risk of a patient or participant getting harmed by a deep brain stimulation performed by a neurosurgeon specialized in deep brain stimulation is lower than that of a pediatric neurosurgeon performing the same intervention. In the case of RCT, a neurosurgeon specialized in deep brain stimulation would be required to carry out the intervention as that type of neurosurgeon is the only neurosurgeon knowledgeable and skilled enough to properly carry out the intervention. Consequently, in a pragmatic RCT, a pediatric neurosurgeon or any other type of neurosurgeon for the matter can perform the intervention as they meet the minimum requirement needed to perform the intervention; that requirement being a neurosurgeon. As it can be seen, pragmatic RCTs are not as low-risk as they are said to be. To make things worse, given the randomization component of pragmatic RCTs, participants are at risk of being paired up with a medical or healthcare professional that may not as much experience carrying out the specific intervention, further increasing the risk of the patient or participant getting harmed.

\section{Disclosure Issues}

The ethical issue from research-practice distinction provides an interest case against researchers against disclosing randomization. As discussed earlier, researchers arguing for disclosure have highlighted that not disclosing randomization is problematic for both ethical and practical reasons, therefore it should be disclosed to participants (Goldstein et al., 2018). Opposing disclosing randomization to patients and participants were researchers that argued that since randomization does not increase risk, disclosing it to participants is not needed. 
In response to the argument made by researchers against disclosing randomization, from analyzing the ethical issue found in researchpractice distinction and the neurosurgical intervention example presented in the previous section, the claim that randomization does not increase risk is false. With research-practice distinction emphasizing the fact that in pragmatic RCTs, not all medical and healthcare professionals are specialized (i.e. not the best individual to carry out the intervention), randomization in pragmatic RCTs does increase risk. The reason for this is because those involved in delivering the intervention in a pragmatic RCT are not all specialized medical and healthcare professionals. As a result, the risk of harm to a patient or participant in a pragmatic RCT increases as given that there is a lower chance that a group of patients or participants is assigned to a specialized medical and healthcare professional, immediately resulting that is a higher chance of the group of patients or participants being at risk of harm in the pragmatic RCT. Therefore, disclosing randomization to participants in a pragmatic RCT is needed as not disclose randomization in a pragmatic RCT would cause an ethical issue as it is wrong to not inform patients and participants participating in a pragmatic RCT of the increased risk of harm resulting from the design of the trial.

\section{Principles and Framework Issues}

In addition to disclosure in pragmatic RCTs, research-practice distinction also impacts pragmatic RCTs in terms of ethical issues is ethical principles, ethical framework and regulatory frameworks with respect to pragmatic RCTs. Traditional ethical framework assumes a clear distinction between research and clinical practice (Horn et al., 2018). With research-practice distinction, traditional ethical framework assumes that since explanatory RCT work under tightly controlled conditions and environments, a thirdparty review is necessary. However, since clinical practice is assumed to be autonomous and selfregulated, a third-part review is not necessary (Horn et al., 2018). In addition, knowing physicianpatient relationships, it prohibits third-party interference. Thus, while it is justified and necessary to have a third-party review in research, it is not justified and necessary to have a third-party in clinical practice. In addition, knowing physicianpatient relationships, it prohibits third-party interference.

When pragmatic RCTs are involved, this becomes a challenge because pragmatic RCTs is for research but realized through interventions routinely used in clinical practice. As mentioned in the literature, the primary underlying ethical issue with pragmatic RCT is that the current ethical principles, ethical framework and regulatory frameworks are not compatible with pragmatic RCT because they are designed based on explanatory RCT. Traditional ethical guidance is more difficult to interpret for researchers, research ethics committees, stakeholders and other decision-makers (Horn et al., 2018). Consequently, the lack of guidance on internationally interpreting accepted research ethics principle in the context of pragmatic RCTs has created a gap that prevents researchers and research ethics committees from effectively analyzing ethical areas within a pragmatic RCTs.

\section{Research Oversight Issues}

The lack of guidance on internationally interpreting accepted research ethics principle in the context of pragmatic RCTs contributes to the concerns surrounding oversight - that oversight delays and hindering the overall progress of research with little added value as expressed by authors and those within the scientific community conducting pragmatic RCTs. One may even argue that the lack of guidance $i$ s the reason why there are delays in the oversight process within pragmatic RCTs. A consequence of having no proper ethical guidance is spending more time determining and analyzing ethical aspects within a pragmatic RCT, resulting in delays in oversight.

\section{Consent and Participation Issues}

As highlighted in the sections earlier, patients and participants, especially those participants that are categorized as vulnerable, in a pragmatic RCT 
are more at risk than people realize. The increased risk is due to two factors: participation selection process (in pragmatic RCTs) and randomization. Given the way that pragmatic RCTs are designed, with there being less strictness in the requirements for participants, this puts vulnerable populations at risk. Instances of vulnerable patients and vulnerable potential participants not being suitable for a study but being capable of enrolling in the study can increase the risk of them being harmed in the study should they end up participating in the study. In turn, this can make them more at risk of harm than non-vulnerable participants, leading to there not being equally low-risk for all participants in the pragmatic RCT.

In terms of randomization, randomization in RCTs combined with the design of pragmatic RCT increases the risk of harm in patients and participants in pragmatic RCTs To make things worse, with there being no unified and systematic method of ethical analysis for pragmatic RCTs, proper oversight on pragmatic RCTs needed to ensure that human subjects are protected from the natural bias of researchers and research institutions in favor of experimentation cannot be done as effectively as it should be. This puts both vulnerable and non-vulnerable participants in pragmatic RCTs at risk. However, given the current issue of inclusion of vulnerable participants in pragmatic RCT, the risk of harm for vulnerable participants becomes worse than the risk of harm for non-vulnerable participants. This leads to the ethical issue of pragmatic RCT not being equally low-risk for all participants.

With the ethical issue of inclusion and vulnerable populations which authors argued for inclusion of vulnerable participants in pragmatic RCT came issues regarding consent of vulnerable populations in pragmatic RCTs. As mentioned in the literature, pragmatic RCT is designed to make the question about vulnerable people and their ability to provide informed consent less relevant when consent is changed. With performing oversight over pragmatic RCTs being a challenge for research ethics committees combined with the other ethical issues (e.g. non-specialized medical and healthcare professionals delivering the intervention), the risk of vulnerable participants being harmed become much higher for them. The level of risk associated with pragmatic RCTs and vulnerable (and non-vulnerable) participants becomes extremely problematic in the cases where it is realized that randomized controlled trials labeled as pragmatic are not actually pragmatic.

\section{Pragmatic Issues}

The pressing ethical issue of pragmatic RCTs not being pragmatic is the most worrisome ethical issue that exists within pragmatic RCTs. The whole purpose of pragmatic RCTs is to be pragmatic through having the pragmatic RCT be conducted in a manner that resembles usual clinical practice and have the results produced from the pragmatic RCT be applicable multiple other settings and not solely the one where the trial was conducted. From the study investigating how pragmatic are randomized controlled trials labeled as pragmatic, it was found that between 1977 to $2017,36 \%$ of RCTs that were on medicines and labeled as pragmatic were not pragmatic. This implies that the $36 \%$ of RCTs labeled as pragmatic were not conducted in a manner that resembled usual clinical practice. Not only does pose problems for the scientific community that will make use of the studies, but also to the participants that have participated in those studies. The ethical issue of pragmatic RCTs not being pragmatic creates two primary problems: one being an ethical issue while the other being a medical ethics issue.

The primary ethical issue that RCTs labeled as pragmatic not being pragmatic creates is that the results produced from such trials will not be applicable multiple other settings and will mainly be applicable to the setting that was used to conduct the trial. The expected consequences produced from this ethical issue include at least one of the following:

- Reevaluating approaches, methods and interventions, which in turn can be time consuming and costly.

- Reconducting experiments and performing trials, which in turn given the situation 
surrounding oversight and pragmatic RCTs, will be time consuming, costly and will unnecessarily deplete of resources.

- Decrease the credibility of pragmatic RCTs, resulting in stakeholders, research ethics committees, practitioners, health administrators, institutions, researchers, bioethicists, and other medical, administrative and healthcare individuals to become skeptic of pragmatic RCTs.

- Legal cases, which can lead to consequences such as the revocation of medical licenses and healthcare licenses.

- Decrease the credibility of researchers that used RCTs labeled as pragmatic but were not being pragmatic in their research.

- Other undesired outcomes not listed above.

The primary medical ethics issue that RCTs labeled as pragmatic not being pragmatic creates is that autonomy, non-maleficence, beneficence and justice are not respected. The expected consequences that can be produced from this medical ethics issue include at least one of the following:

- Harm to patients and/or participants.

- Death of patients and/or participants.

- Distrust and disapproval from the public toward pragmatic RCTs, researchers and/or institutions.

- Decrease the credibility of pragmatic RCTs, resulting in patients and potential participants become skeptic of pragmatic RCTs.

- Other undesired outcomes not listed above.

As autonomy requires patients and participants to be able to think, decide and act on one's own free initiative, which one's thoughts, decisions and actions are heavily influenced by the information provided to them, autonomy is not respected as patients and participants thoughts, decisions and actions were subject to misinformation. By not providing accurate information to the patients and participants, this violates beneficence as promoting what is best for the patients and participants no longer takes place. In order to promote what is best for the patients and participants, it is key that they are properly informed about the activities that they will undertake in the experiment, the risks associated with the experiment and intervention, the benefits from participating in the study, and other crucial and necessary information needed to allow patients and participants to make a well informed decision that is in their best interest.

Non-maleficence is not respected as with RCTs labeled as pragmatic not being pragmatic, those RCTs are no longer considered as low-risk trials. As a result, the risk of harm towards patients and participants in such trials are increased to either a degree equivalent to explanatory RCTs or higher. The way the risk of harm towards patients and participants in such trials is increased to a degree higher than equivalent to explanatory RCTs is through cases where certain aspects of pragmatic RCTs are followed in the RCTs labeled as pragmatic (but are not pragmatic). For instance, if the RCT followed the intervention delivery of that of pragmatic RCT, then essentially instead of the RCT having specialized medical professionals deliver the intervention, you would have nonspecialized medical and healthcare professionals conduct an explanatory RCT. This is an extremely risky approach that leaves patients and participants at high risk as given that the non-specialized medical and healthcare professionals are not knowledgeable and experienced enough to carry out interventions in an explanatory RCT (compared to that of a specialized medical professional), there is a high chance that participants in the trial will be harmed.

Whether or not participants get harmed in the process, the results produced from the study can lead to harm to patients that undergo the intervention. A reason for that would be because of the fact that the intervention was not conducted in a setting that was similar to that used in the RCT. As a result, not only is non-maleficence violated, but justice is also violated as well because fairness is not respected. It is not fair that some patients and participants are more at risk than others. In addition, since patients and participants in the RCT labeled as pragmatic but is not pragmatic were 
misinformed about what they were getting themselves, their right of being provided correct and accurate information about the trial prior to signing up for it.

\section{Conflicts of Interest}

In starting off the discussion on conflicts of interest in pragmatic RCTs, we will first define conflicts of interest from a medical perspective, discuss relevant information mentioned within the literature, highlighting and elaborating on where conflicts of interest can occur within a pragmatic RCT. From there, we will investigate conflicts of interest in clinical practice and discuss the correlation between those conflicts of interest cases and conflicts of interest in pragmatic RCTs, then conclude with arguing that not only does conflicts of interest occur within pragmatic RCTs, but also describe the lethality of consequences that could potentially be produced by conflicts of interest in pragmatic RCTs.

Conflicts of interest is when an individual has interests in the outcome of the research that may lead to personal advantage and that might therefore, in actuality or appearance, compromise the integrity of the research (Gorman, 2018). Within medical practice, conflicts of interest occur when the interests of clinicians do not align with the interest of their patients (Tonelli, 2007). Within medical research, conflicts of interest occur when the interests of the researchers do not align with the interest of the participants. From the current situation in pragmatic RCTs, there are two ethical issues that either a direct or indirect correlation with conflicts of interest. The first ethical issue that has a direct correlation with conflicts of interest pertains to the oversight process performed by research ethics committees. We know that the committees are responsible for overseeing research ethics, grants and contracts, conflicts of interest, patient safety and pharmacy within a study. We also know that there is no ethical framework in place to address the ethical components of a pragmatic RCT. As a result, research ethics committees are not properly able to access ethical issues in pragmatic RCTs. The direct correlation between this ethical issue and conflicts of interest is that one of those aspects of research that ethics committees are supposed to oversee is conflicts of interest. With no solid ethical framework for pragmatic RCTs, interests of healthcare researchers and providers that do not align or follow ethical principles can more easily be carried out in a pragmatic RCT. This implies that within pragmatic RCTs, conflicts of interest can arise prior to the oversight process.

The second ethical issue which has an indirect correlation with conflicts of interest pertains to how pragmatic are RCTs labeled as 'pragmatic'. From the literature and discussion on the ethical issue from earlier, we know that between 1977 to $2017,36 \%$ of RCTs that were on medicines and labeled as pragmatic were not pragmatic. This implies that those RCTs were explanatory RCTs, which it is known to professionals and even the public that conflicts of interest exists within explanatory RCTs. Two examples of researches that use explanatory RCT and have conflicts of interest is intensive care research and oncology medication research. A study from Michael Darmon et al. that studied time trends in the reporting of conflicts of interest, funding, and affiliation with industry in intensive care research found that from the 374 studies that they evaluated, conflicts of interest statements was available in $65 \%$ of the studies and $8 \%$ had declared conflicts of interest. Their study suggested that conflicts of interest reporting have been unreliable before the International Committee of Medical Journal Editors (ICMJE) statements (Darmon et al., 2018). A study by Cole Wayant et al. studied financial conflicts of interest among oncologist authors and clinical drug trials studied 1007 authors of whom 344 oncologist authors from 43 published trials were included in their study. The findings from there study found that 263 oncologist-authors $(76.5 \%)$ received at least 1 industry payment. The median value of general payments to the 344 oncologist authors was $\$ 2,828$ and the median value of associated research payments was $\$ 164,644$. Cumulatively, the 344 oncologist authors received a total of $\$ 216,627,353$ (Wayant et al., 2018). In the discussion section of their research, they mention how their study found that 
financial relationships between oncologist athors and the pharmaceutical industry may be common, expensive, and frequently undisclosed (Wayant et al., 2018). From the two examples alone, we can deduce that conflicts of interest do in fact exist within explanatory RCTs. With RCTs labeled as pragmatic but actually being explanatory, as those RCTs have been passed as pragmatic RCTs and given that conflicts of interest does occur within explanatory RCTs, therefore there are conflicts of interest in pragmatic RCTs.

There are many consequences that can be produced from the conflicts of interest in pragmatic RCTs. Consequences can include one or more of the following:

- Harm to patients and/or participants.

- Death of patients and/or participants.

- Legal cases, which can lead to consequences such as the revocation of medical licenses and healthcare licenses.

- Decrease the credibility of pragmatic RCTs, resulting in stakeholders, research ethics committees, practitioners, health administrators, institutions, researchers, bioethicists, and other medical, administrative and healthcare individuals to become skeptic of pragmatic RCTs.

- Distrust and disapproval from the public toward pragmatic RCTs, researchers and/or institutions.

- Other undesired outcomes not listed above.

\section{Discussion}

Through investigating literature on pragmatic RCTs and performing a comprehensive ethical analysis on pragmatic RCTs, we find that there many ethical issues and concerns that stem from both the design and the use of pragmatic RCTs. The main ethical and medical ethics issue with the design of pragmatic RCTs is that they pose a threat to participants, more specifically the vulnerable participants.

The main ethical and medical ethics issue with the use of pragmatic RCTs is that participants in the trial are more at risk of harm, making pragmatic RCTs not a low-risk trial and violating beneficence and non-maleficence; the mislabeling of explanatory RCTs as pragmatic RCTs will lead to results that cannot be replicated in different settings, violating justice; and the lack of inclusion and non-strict eligibility requirements in pragmatic RCTs causes vulnerable patients and participants to be more at risk of harm than non-vulnerable patients and participants, resulting in pragmatic RCTs not being equally low-risk for all participants. The new ethical issue that has been discussed in pragmatic RCTs are conflicts of interest. Whether it is financial conflicts of interest or some other form of conflict of interest, conflicts of interest does occur within pragmatic RCTs. They either occur through the genuine pragmatic RCTs themselves or through the RCTs labeled as pragmatic but are in fact explanatory.

\section{Conclusion}

While pragmatic RCT is a useful study design that allows medical and healthcare researchers and professionals to better the effectiveness of specific interventions in usual clinical conditions and dayto-day practice, there are ethical issues, medical ethics issues and concerns associated with the design and application of it. To address these issues and concerns, it is necessary that a new set of ethical principles are developed along with the creation of an ethical framework either dedicated to or compatible with pragmatic RCTs. The ethical framework should be developed with inclusion in mind. In addition, creation of policies to safeguard and protect vulnerable patients and participants should be developed and enforced to ensure the protection and safety of the vulnerable patients and participants within research utilizing pragmatic RCTs.

With conflicts of interest being likely to become more challenging in the years to come (Bauchner, Fontanarosa \& Flanagin, 2018), it is critical that guidelines are made to allow for better handling of conflicts of interest in pragmatic RCTs. It is extremely important that editors of scientific journals successfully complete their responsibilities, which includes ensuring that all 
published information are accurate and objective and to maintaining the integrity of the scientific record. In addition, it is critical that authors report conflicts of interest information accurately, completely, and transparently so readers can evaluate whether the information in the article could be biased because of the author's potential conflict of interest (Bauchner, Fontanarosa \& Flanagin, 2018).

\section{References}

Baucher H., Fontanarosa P. B. \& Flanagin A. (2018). Conflict of Interest, Authors, and Journals. American Medical Association. 320. 22. 2317.

Califf R. M. \& Sugarman, J. (2015). Exploring the Ethical and Regulatory Issues in Pragmatic Clinical Trials. Clinical Trials. 12. 5. 436-441.

Charlton B. G. (1994). Understanding Randomized Controlled Trials: Explanatory or Pragmatic? Oxford University Press. 11. 3. 243 244.

Colli A., Pagliaro L. \& Duca P. (2014). The Ethical Problem of Randomization. Internal and Emergency Medicine. 9. 801 - 802.

Dal-Ré R., Janiaud P. \& Loannidis P. A. (2018). Real World Evidence: How Pragmatic are Randomized Controlled Trials Labeled as Pragmatic. BMC Medicine. 16. 49. 1 - 3.

Darmon M., Helms J., De Jong A., Hjortrup P. B., Weiss E., Granholm A., Pinciroli R., Poussardin C., Petersen M. W., Sigaut S., Barreto B. B., Moller M. H. \& Azoulay E. (2018). Time Trends in the Reporting of Conflicts of Interest, Funding and Affiliation with Industry in Intensive Care Research: A Systematic Review. Intensive Care Medicine. 44. 1672 -1677 .

Emanuel E. J., Crouch R. A., Arras J. D., Moreno J. D. \& Grady C. (2003). Ethical and Regulatory Aspects of Clinical Research
- Readings and Commentary. The Johns Hopkins University Press. 103 - 107.

Fries J. F. \& Krishnan E. (2004). Equipoise, Design Bias, and Randomized Controlled Trials: The Elusive Ethics of New Drug Development. Arthritis Research \& Therapy. 6. 3. 250-251.

Goldstein C.E., Weijer C., Brehaut J. C., Fergusson D. A., Grimshaw J. M., Horn A. R. \& Taljaard M. (2018). Ethical Issues in Pragmatic Randomized Controlled Trials: A Review of the Recent Literature Identities Gapes in Ethical Argumentation. BMC Medical Ethics. 19. 14. $1-10$.

Gorman D. M. (2018). Can We Trust Findings of Intervention Research? The Role of Conflicts of Interest. Prevention Science. 19. 3. 295 - 297.

Government of Canada (2018). Tri-Council Policy Statement: Ethical Conduct for Research Involving Humans. Retrieved on April 17, 2019 from:

$$
\begin{aligned}
& \text { www.pre.ethics.gc.ca/eng/policy- } \\
& \text { politique/initiatives/tcps2- } \\
& \text { eptc2/chapter9-chapitre9/\#toc09-1 }
\end{aligned}
$$

Horn A. R., Weijer C., Grimshaw J., Brehaut J., Fergusson D., Goldstein C. E. \& Taljaard M. (2018). An Ethical Analysis of the SUPPORT Trial: Addressing Challenges Posed by Pragmatic Comparative Effectiveness Random Controlled Trial. John Hopkins University Press. 28.1. 86 -111 .

MacPherson H. (2004). Pragmatic Clinical Trials. Complementary Therapies in Medicine. 136 140.

Patsopoulos N. A. (2011). A Pragmatic View on Pragmatic Trials. Dialogues in Clinical Neuroscience. 13.2. 217 - 220.

Sidani S. (2015). Health Intervention Research - Understanding Research Design \& Methods. Sage. $54-60,101-106$.

Tonelli, M. R. (2007). Conflicts of Interest in Clinical Practice. Chest. 132. 2. $664-669$. 
Wayant C., Turner E., Meyer C., Sinnet P. \& Vassar M. (2018). Financial Conflicts of Interest Among Oncologist Authors of Reports of Clinical Drug Trials. American Medical Association. 4. 10. 1426 - 1428.

Welch M. J., Lally R., Miller J. E., Pittman S., Brodsky L., Caplan A. L., Uhlenbrauk G., Louzao D. M., Fischer J. H. \& Wilfond B. (2015). The Ethics and Regulatory Landscape of Including Vulnerable Populations in Pragmatic Clinical Trials. Clinical Trials. 12. 5. $503-510$.

Wenner D. M. (2016). Barriers to Effective Deliberation in Clinical Research Oversight. HEC Forum. 28. 245 - 246. 